\title{
Analysis of Olig2 and YKL-40 expression: a clinicopathological/ immunohistochemical study for the distinction between subventricular zone II and III glioblastomas
}

\author{
Kelvin Manuel Piña Batista ${ }^{1}$, Bruno Augusto Lourenco Costa ${ }^{2}$, Isabel Cuervo-Arango Herreros ${ }^{1}$, \\ Ivan Fernandez Vega ${ }^{3}$, Julio Cesar Gutierrez Morales ${ }^{1}$, Aitana Vallina Alvarez ${ }^{4}$, Pablo Isidro Marron ${ }^{4}$, \\ Ángela Meilán ${ }^{5}$, Aurora Astudillo ${ }^{3}, K_{\text {Kenia Yoelvi Alvarez }}{ }^{6}$ \\ ${ }^{1}$ Department of Neurosurgery, Hospital Universitario Central de Asturias, ${ }^{2}$ Department of Neurosurgery, Hospitais da Universidade \\ de Coimbra, ${ }^{3}$ Department of Neuropathology, Hospital Universitario de Araba, ${ }^{4}$ IUOPA biobank, Hospital Universitario Central \\ de Asturias, ${ }^{5}$ Department of Neuroradiology, Hospital Universitario Central de Asturias, ${ }^{6}$ Hospital Álvarez Buylla, Asturias, Spain
}

\begin{abstract}
Glioblastomas (GBS) are the most common and lethal primary brain tumors in the adults. Glioblastomas originates either from astrocytes that have accumulated mutations and de-differentiated or from neural stem cells within the subventricular zone (SVZ) in close contact with the vasculature. Recently, several studies have hypothesized that gliomagenesis occurs in perivascular niches with highly invasive peripheral proliferating zones. The purpose of our study was to investigate the pathological and clinical significance of Olig2 and YKL4O immunoexpression in 152 GBS in relationship to the SVZ II and III. Olig2 expressions were successfully detected in 12 (15.58\%) of 77 SVZ type II GBS and 16 (21.3\%) of 75 SVZ type III GBS, respectively. YKL-40 expression was observed in 45 (58.4\%) of 77 SVZ type II GBs and in 17 (22.6\%) of 75 SVZ type III GBs, respectively. Stepwise multivariate Cox proportional hazards models were used, and the prognostic factors to significantly impact OS were: PFS < 54 weeks (HR: 5.86; Cl: 3.02-11.33; $p=0.00$ ); radiotherapy (HR: 0.34; Cl: $0.18-0.60 ; p=0.00$ ); radio- and chemotherapy ( $H R: 0.05 ; C l: 0.03-0.10 ; p=0.0$ ), and YKL-40+ GBS (HR: $1.61 ; C l: 1.28-2.31 ; p=0.01)$.
\end{abstract}

Key words: glioblastoma, subventricular zone, Olig2, YKL-40, clinicopathological, immunohistochemical.

\section{Introduction}

Glioblastoma (GB) is the most common primary brain tumor in the adults. Median survival of GB patients treated with aggressive multimodal therapy, including total resection, combined radiation and chemotherapy, and adjuvant chemotherapy is about
12 months $[9,18,24,25]$. Glioblastoma is actually considered a heterogeneous and dynamic disease $[13,17,21]$. Recent studies demonstrate that GB originates either from astrocytes that have accumulated mutations and de-differentiated or from neural stem cells within the subventricular zone (SVZ) in close 
contact with the vasculature. In animal models, those neural stem cells may play a role in the gliomagenesis, recurrence, and resistance to therapies $[17,28]$. Probably, survival may be strongly affected by the tumor's relationship to the SVZ. Based on the MRI spatial relationship of the GB, Lim et al. [21] distinguish 4 types: type I, tumor contacting the SVZ and infiltrating cortex; type II, tumor contacting the SVZ only; type III, tumor involving the cortex but not involving the SVZ, and type IV, tumors spare both the cortex and the SVZ.

The precise origin of glioma stem cells (GSCs) is still unclear. Recent research efforts hypothesize that gliomagenesis occurs in perivascular niches with highly invasive peripheral proliferating zones $[6,12,29]$. Several studies report that Olig2 expression is limited in GSCs $[7,12,22]$ and is probably being related to the SVZ type II as a proliferation regulator and glioma progenitor cell marker. Olig2 (a basic helix-loop-helix transcription factor) is expressed in the postnatal SVZ and plays a critical role in the lineage specification of progenitor cells into neurons and oligodendrocytes [20]. However, the literature on Olig2 and its association with glioblastoma prognosis is ambivalent $[1,11,12,22]$. Recent reports have found associations between glioblastoma and neural stem cells expressing Olig2 [8,12,20,35]. Therefore, a significant amount of the ongoing GB research is focused on better understanding how cells expressing Olig2 contribute to the gliomagenesis and therapeutic targets.

YKL-40 (also known as CHI3LI), a member of mammalian chitinase-like protein, is a growth factor for connective tissue cells that (although its function is not well defined) may play a role in the migration of endothelial cells, inflammation and tissue remodeling. It is also overexpressed in glioblastoma compared to anaplastic gliomas and low-grade gliomas [26,30,36].

The localization of YKL-40 expression related to the SVZ remains unclear. Given that YKL-40 immunoexpression is associated with poor prognosis and Olig2 is linked to the neural stem/progenitor cells, we investigated the clinical/prognostic significance of YKL-40 and Olig2 expression related to SVZ type II/III GBs.

\section{Material and methods Patients and samples}

A retrospective study was performed on 152 patients harboring GBS in the SVZ type II/III treat- ed by subtotal resection between 2006 and 2010 . Paraffin-embedded samples were obtained from the Biobank of Asturias, in Central of Asturias University Hospital, Spain. For each case, the hematoxylin-eosin sections were reviewed and all cases were classified according to the World Health Organization (WHO) classification system as glioblastoma multiforme by a senior neuropathologist. Detailed data regarding clinical presentation, pathological analysis, progression-free survival, and overall survival outcome were recorded. Tumors were classified as limited to the cortex or type II (77 GBS) or limited to the SVZ or type III (75 GBs). Both, YKL-40 and Olig2 expressions were investigated by immunohistochemistry in all of the aforementioned cases. All samples used in this study were obtained with the approval of the Committee for Ethical Review Board of Central of Asturias University Hospital.

The extent of resection was determined on the basis of MRI results (within 48 hours after surgery). Subtotal and total resection were defined as those tumors with residual and no residual enhancement, respectively, achieved by comparing pre- and postoperative MRI. The extent of resection was classified as total (>95\%), subtotal (<95\%) or biopsy by a neuroradiologist blinded to patient outcomes. Patients with a Karnofsky Performance Scale (KPS) score $\geq 70$ and age $<60$ were included to receive conventional radiotherapy and chemotherapy after surgical resection: 1.8-2.0 Gy per day, over a period of 6 weeks, for a total dose of 60 Gy and temozolomide therapy at a dose of $75 \mathrm{mg} / \mathrm{m}^{2}$ per day, seven days a week for 42 consecutive days during radiotherapy (as used in the EORTC study by Stupp et al.) $[19,31,33]$.

\section{Immunohistochemistry}

Monoclonal antibodies for YKL-40 (ab86428; Abcam, Cambridge, UK; dilution 1 : 500), and polyclonal anti-Olig2 antibody (ab9610; EDM Millipore, Massachusetts, USA; dilution 1 : 500) were used. Five-micrometer consecutive sections were cut from the paraffin-embedded samples. Each tissue section was deparaffinized and rehydrated with graded ethanol. Antigen retrieval was accomplished by boiling the sections for 15 minutes in $10 \mathrm{mmol} / \mathrm{EDTA}$, $\mathrm{pH}$ 6.0. Endogenous peroxidase activity was blocked with a $3 \%$ hydrogen peroxide for 10 minutes. Then, slides were incubated overnight at $4^{\circ} \mathrm{C}$ with respective primary antibodies. Visualization was performed using DAB (3,3'-diaminobenzidine). Tissue sections 
were counterstained with hematoxylin, dehydrated, and mounted. An oligodendroglioma and hepatocellular carcinoma with immunoreactivity was used for positive control (Olig2 and YKL-40, respectively). As a negative control the primary antibodies were omitted.

Immunoreactivity for YKL-40 was evaluated by a three-tiered system ( 0 - negative; 1 - moderate/ patchy staining in tumor cell; 3 - strongly positive) [26]. Staining for Olig2 was scored only in cells as positive (1), and negative or weak positive (0). The authors did the scoring independently. To obtain more accurate results, 2 independent observers evaluated all immunostaining experiments.

\section{Statistical analysis}

All statistical analyses were performed with SPSS Statistics version 20 (IBM) with a significance level of $5 \%(p \leq 0.05)$. The $\chi^{2}$ test and the Fisher's exact test were used for the evaluation of the association between Olig2 and YKL-40 (positive vs. negative immunoexpression) and covariates. Karnofsky (KPS) from 3 months was included for analyses because at this time used to occur the most significant and lasting change in patient clinical status. Overall survival (OS) was determined from the date of diagnosis to the date of death. Progression-free survival (PFS) was determined from the date of diagnosis to the date of relapse. Kaplan-Meier method was used to investigate Olig2 and YKL-40 expression as univariate in prediction of the patient's survival related to SVZ. Multivariate survival analyses were performed by a stepwise Cox proportional hazards model used for univariate and multivariate analyses of PFS and OS.

\section{Results}

Patient demographics are presented in Table I. Expressions of Olig2 and YKL-40 in 152 GBs were investigated by IHC. Olig2 expressions were successfully detected in 12 (15.58\%) of 77 SVZ type II GBS and $16(21.3 \%)$ of 75 SVZ type III GBs, respectively. YKL-40 expression was observed in 45 (58.4\%) of 77 SVZ type II GBs and in 17 (22.6\%) of 75 SVZ type III GBs, respectively. Positive expression of YKL-40 was found in the cytoplasm of GB tumor cells (Fig. 1). Olig2+ GB cells showed strong nuclear immunoreactivity. For better understanding of the analytical results, YKL-40 expressions were classified as positive $(1+, 2+)$ or negative $(0)$.

\section{Relationship between the immuno- expression of Olig2 and YKL-40 and clinico-pathological findings}

The results of the pathologic findings are shown in Table II. Expression of Olig2 was not associated with the patient's age ( $\leq 65$ years old versus $>65$ years old), gender, Karnofsky at diagnosis (KPS at $D x)$, progression-free survival (PFS), overall survival (OS), and GB type. However, Olig 2 was associated with KPS at 3 months $(p=0.035)$. YKL-40 did not $(p>0.05)$ correlate with gender, and KPS at diagno-

Table I. Clinical characteristics of 152 patients with glioblastoma

\begin{tabular}{|c|c|}
\hline Groups & Characteristics \\
\hline Age & Years \\
\hline$\leq 65$ & 93 \\
\hline$>65$ & 59 \\
\hline Gender & $N^{0}$ \\
\hline Male & 79 \\
\hline Female & 73 \\
\hline Karnofsky (KPS) at Dx, Score & $N^{\circ}$ \\
\hline$<70$ & 20 \\
\hline$\geq 70$ & 132 \\
\hline KPS at 3 months, Score & $N^{\circ}$ \\
\hline$<70$ & 47 \\
\hline$\geq 70$ & 105 \\
\hline Overall Survival, OS & Weeks \\
\hline$\leq 54$ & 98 \\
\hline$>54$ & 53 \\
\hline Progression-free survival, PFS & Weeks \\
\hline$\leq 54$ & 131 \\
\hline$>54$ & 21 \\
\hline Subventricular relationship & $N^{0}$ \\
\hline Group II & 77 \\
\hline Group III & 75 \\
\hline Extent of tumor resection & $\mathrm{N}^{\circ}$ \\
\hline Gross total resection & 40 \\
\hline Subtotal resection & 86 \\
\hline Biopsy & 26 \\
\hline First-line therapy & $\mathrm{N}^{0}$ \\
\hline Radiotherapy & 26 \\
\hline Chemotherapy & 1 \\
\hline Radiotherapy and chemotherapy & 98 \\
\hline Therapeutic abstention & 27 \\
\hline
\end{tabular}



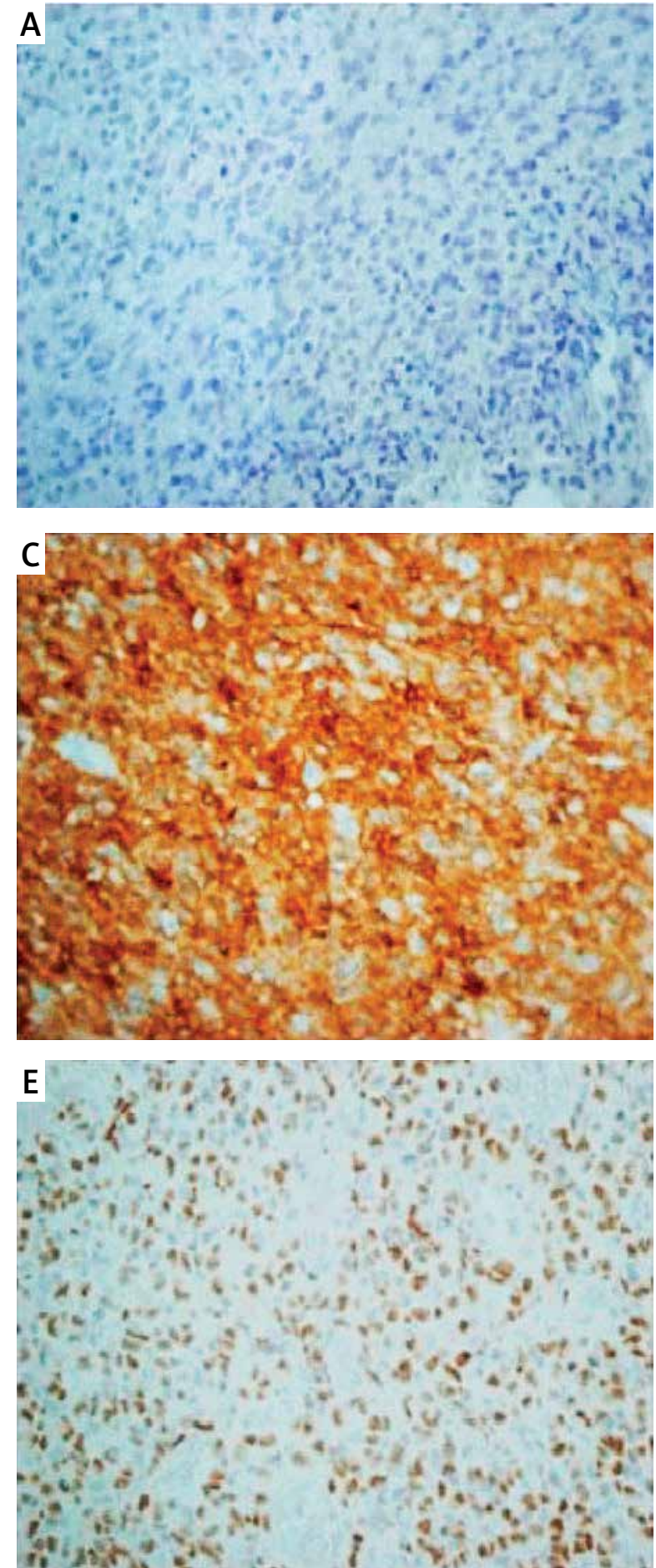

sis. Interestingly, YKL-40 was significantly associated with age, KPS at 3 months, PFS, OS, and GB type. Kaplan-Meier analysis showed that YKL-40 (CHI3L1) expression was significantly different from Olig2 expression ( $p>0.05$ ) (Figs. 2 and 3).
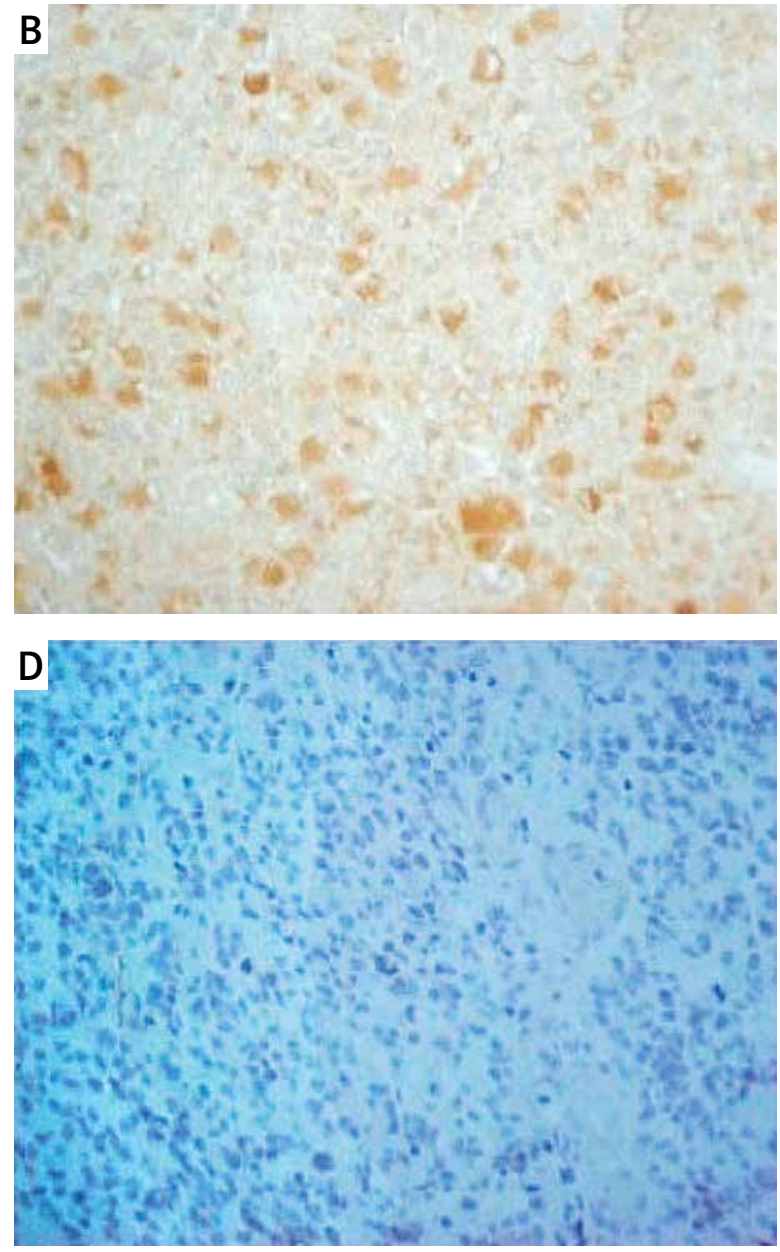

Fig. 1. Examples of negative and positive staining are shown for YKL-40 and Olig2. A) Example of negative staining (-) tumor cells for YKL-40. B) Patchy staining (+/-) for YKL-40. C) Strong staining (+) for YKL-40. D) Tumor sample with negative staining $(-)$ for Olig2. E) Positive expression for Olig2 (+) (200× magnification).

\section{Univariate and multivariate analysis of prognostic variables}

To identify and evaluate the variables with potential prognostic significance in patients with $\mathrm{GB}$, univariate and multivariate analysis using Kaplan-Meier 
Table II. Correlations between CHI3L1 and Olig2 expression in tumor tissues and clinic pathological features of patients with glioblastoma

\begin{tabular}{|c|c|c|c|c|c|c|}
\hline \multirow[t]{2}{*}{ Variables } & \multicolumn{2}{|c|}{ CHI3L1 } & \multirow[t]{2}{*}{$p$ value } & \multicolumn{2}{|c|}{ Olig2 } & \multirow[t]{2}{*}{$p$ value } \\
\hline & Positive & Negative & & Positive & Negative & \\
\hline \multicolumn{7}{|l|}{ Age } \\
\hline$<65$ & 31 & 62 & $0.019^{*}$ & 20 & 73 & 0.218 \\
\hline$>65$ & 31 & 28 & 8 & 51 & & \\
\hline \multicolumn{7}{|l|}{ Gender } \\
\hline Male & 34 & 45 & 0.557 & 13 & 66 & 0.516 \\
\hline Female & 28 & 45 & 15 & 58 & & \\
\hline \multicolumn{7}{|l|}{ KPS at Dx } \\
\hline$<70$ & 11 & 9 & 0.165 & 2 & 18 & 0.297 \\
\hline$>70$ & 51 & 81 & 26 & 106 & & \\
\hline \multicolumn{7}{|l|}{ KPS at 3 months } \\
\hline$<70$ & 26 & 21 & $0.015^{\star}$ & 4 & 43 & $0.035^{*}$ \\
\hline$>70$ & 36 & 69 & 24 & 81 & & \\
\hline \multicolumn{7}{|l|}{ PFS } \\
\hline$<54$ & 58 & 73 & $0.013^{*}$ & 22 & 109 & 0.157 \\
\hline$>54$ & 4 & 17 & 7 & 14 & & \\
\hline \multicolumn{7}{|l|}{ OS } \\
\hline$<54$ & 52 & 46 & $0.00^{*}$ & 15 & 83 & 0.164 \\
\hline$>54$ & 10 & 44 & 13 & 41 & & \\
\hline \multicolumn{7}{|l|}{ GBMs } \\
\hline SVZ II & 45 & 32 & $0.00^{*}$ & 12 & 65 & 0.361 \\
\hline SVZ III & 17 & 58 & 16 & 59 & & \\
\hline \multicolumn{7}{|l|}{ Extent of tumor resection } \\
\hline Gross total resection & 9 & 31 & $0.02^{\star}$ & 9 & 31 & 0.281 \\
\hline Subtotal resection & 36 & 50 & 17 & 69 & & \\
\hline Biopsy & 17 & 9 & 2 & 24 & & \\
\hline \multicolumn{7}{|l|}{ First-line therapy } \\
\hline Radiotherapy & 14 & 12 & $0.029^{*}$ & 1 & 26 & $0.023^{*}$ \\
\hline Chemotherapy & 0 & 1 & 1 & 0 & & \\
\hline Radiotherapy and chemotherapy & 32 & 66 & 22 & 76 & & \\
\hline Therapeutic absention & 16 & 11 & 1 & 26 & & \\
\hline
\end{tabular}

$\chi^{2}$ tests, ${ }^{*} p<0.05$

and Cox proportional hazard model was performed. In a univariate proportional hazards regression analysis, the factors associated with survival were: age, KPS at 3 months, YKL-40 (CHI3L1), and Olig2. In addition, univariate analysis confirmed that neither the gender $(p=0.13)$ nor Olig2 immunoexpression $(p=0.86)$ affected OS.
We evaluated the potential prognostic factors following the Cox proportional hazards models using forward stepwise multivariate selection analysis (Table III). The variables significantly associated with OS were: PFS < 54 weeks (HR: 5.86; Cl: 3.02$11.33 ; p=0.00$ ); radiotherapy (HR: $0.34 ; \mathrm{Cl}: 0.18-0.60$; $p=0.00$ ); radio- and chemotherapy (HR: 0.05 ; $\mathrm{Cl}: 0.03$ - 


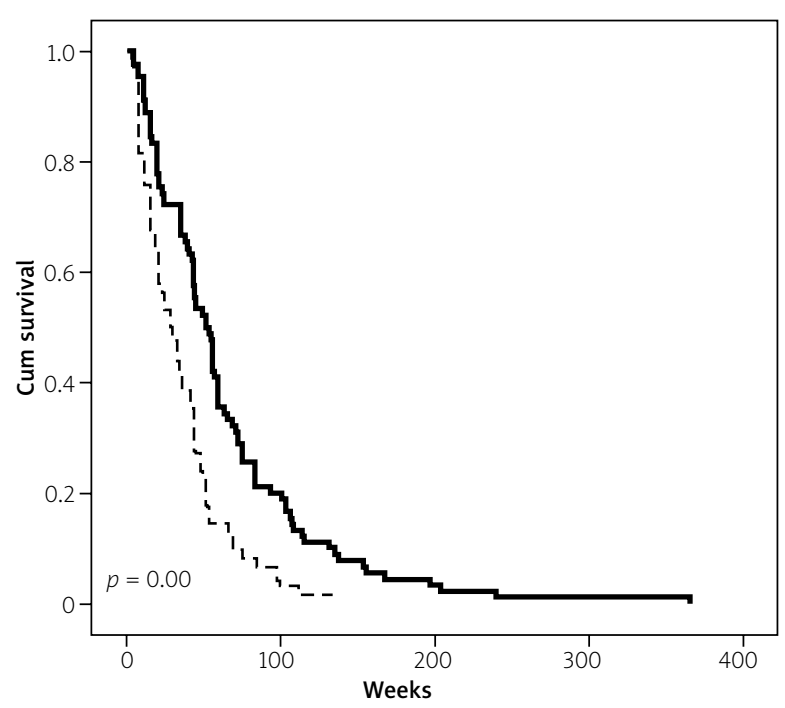

$\boldsymbol{\sim}$ Negative CHI3L1 expression - - I Positive CHI3L1 expression

Fig. 2. Patients expressing YKL-40 (CHI3L1) in tumor tissues show a significantly worse overall survival. Kaplan-Meier analysis for overall survival in a series of glioblastomas according to YKL 40 expression.

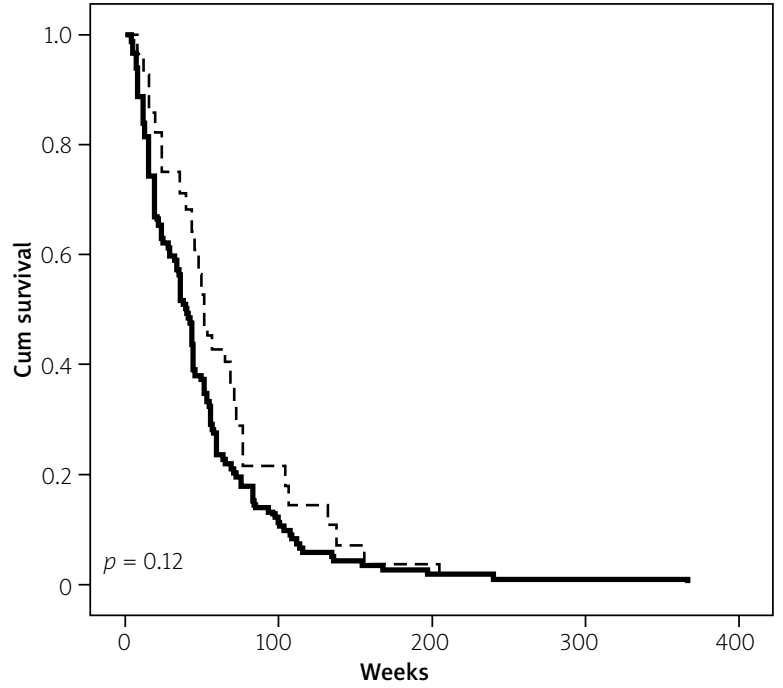

Negative Olig2 expression _ _ r Positive Olig2 expression

Fig. 3. Kaplan-Meier overall survival curves of the series of 152 glioblastomas with a different expression of Olig2. The difference in survival between Olig2+ and Olig2- is not statistically significant $(p=0.12)$.

Table III. Univariate and multivariate analyses of variables associated with survival

\begin{tabular}{|c|c|c|c|c|c|c|}
\hline \multirow[t]{2}{*}{ Variables } & \multicolumn{3}{|c|}{ Univariate analysis } & \multicolumn{3}{|c|}{ Multivariate analysis } \\
\hline & $\mathrm{HR}$ & $95 \% \mathrm{Cl}$ & $p$ value & HR & $95 \% \mathrm{Cl}$ & $p$ value \\
\hline Age, years (< 65 vs. > 65) & 0.46 & $0.33-0.65$ & $0.00^{*}$ & 0.76 & $0.52-1.12$ & 0.17 \\
\hline Gender (male vs. female) & 1.28 & $0.92-1.78$ & 0.13 & 1.21 & $0.85-1.71$ & 0.27 \\
\hline KPS at Dx (<70 vs. > 70) & 0.42 & $1.46-3.82$ & $0.00^{*}$ & & & \\
\hline KPS at 3 months (< 70 vs. $>70)$ & 3.95 & $2.72-5.73$ & $0.00^{*}$ & 1.50 & $0.97-2.32$ & 0.63 \\
\hline PFS, weeks (< 54 vs. > 54) & 0.13 & $0.07-0.25$ & $0.00^{*}$ & 5.41 & $2.78-10.54$ & $0.00^{*}$ \\
\hline CHI3L1-GBM cells (pos. vs. neg.) & 1.22 & $1.08-1.38$ & $0.00^{*}$ & 1.61 & $1.12-2.31$ & $0.01^{*}$ \\
\hline Olig2-GBM (pos. vs. neg.) & 0.98 & $0.84-1.15$ & 0.86 & & & \\
\hline Biopsy & & & $0.00^{*}$ & & & \\
\hline Gross total resection & 0.23 & $0.14-0.40$ & $0.00^{*}$ & & & \\
\hline Subtotal resection & 0.31 & $0.19-0.49$ & $0.00^{*}$ & & & \\
\hline Radiotherapy & & & $0.00^{*}$ & 0.34 & $0.18-0.60$ & $0.00^{*}$ \\
\hline Chemotherapy & 2.65 & $0.35-20.1$ & 0.34 & 1.02 & $0.13-8.02$ & 0.97 \\
\hline Radiotherapy and chemotherapy & 0.10 & $0.05-0.17$ & $0.00^{*}$ & 0.05 & $0.03-0.10$ & $0.00^{*}$ \\
\hline Therapeutic absention & 2.84 & $1.59-5.04$ & $0.00^{*}$ & & & \\
\hline
\end{tabular}

Fisher's exact, * $p<0.05$

KPS - Karnofsky score, KPS at Dx - Karnofsky score at diagnosis, PFS - progression-free survival

$\mathrm{Cl}$-confidence interval, HR - hazard ratio, pos. - positive, neg. - negative 
$0.10 ; p=0.0)$, and YKL-40+GBs (HR: 1.61; Cl: 1.28-2.31; $p=0.01)$. However, Olig2+ GBs were not included in multivariate analysis.

\section{Discussion}

Although the SVZ has been recognized for many years, its neurogenesis and gliomagenesis remains unclear. Subventricular zone may be a source of tumors with higher proliferative and invasive capacities [5]. Recent research efforts in neuro-oncology are focused in targeting the tumorigenesis theory and to find signal transduction pathways that influence the GB development and change its dismal prognosis, given that surgery and adjuvant chemotherapy with radiotherapy are insufficient due to a diffuse infiltration by tumor tissue into the brain $[4,12,37]$. Therefore, identifying molecular targets that could provide prognostic new data is needed and would be helpful for its therapy.

Olig2+ GBs are not significantly expressed in GB type II and III, against predictive and critical functions for Olig2 [32,34]. Under the stem cell hypothesis, Olig2 fulfills criteria of a lineage-restricted competence factor for gliogenesis $[8,15]$ that is necessary for the development of neural progenitors and progeny cells in the CNS [12]. Like others, [22] we have suggested that Olig2 expression may be downregulated in mature astrocyte.

YKL-40 is a potent angiogenic factor that was recently identified to be one of the most expressed proteins in GB when compared to low-grade glioma and normal brain. YKL-40 protein expression was proposed as a potential serum marker for $G B$ $[10,16,26]$. High YKL-40 expression in GB has been correlated with a short OS and a poor response to radiotherapy $[14,16]$. We found that GBs contacting the SVZ trended with shorter OS, although it is unclear if tumors contacting the SVZ have more aggressive behavior, allowing tumor stem cells and their progeny to rapidly proliferate and migrate. The main reasons for a less favorable outcome in GB patients with SVZ involvement are not yet completely understood. Interestingly, we have found a significantly greater YKL-40 expression among the subventricular zone contacting GBs than Olig2+ GBs. Previous work [27] has determined that YKL-40 was particularly linked to SVZ type IV and V. One of the limitations of this study was the small sample size of SVZ type II patients. According to our results, there was also a trend toward a worse OS among SVZ
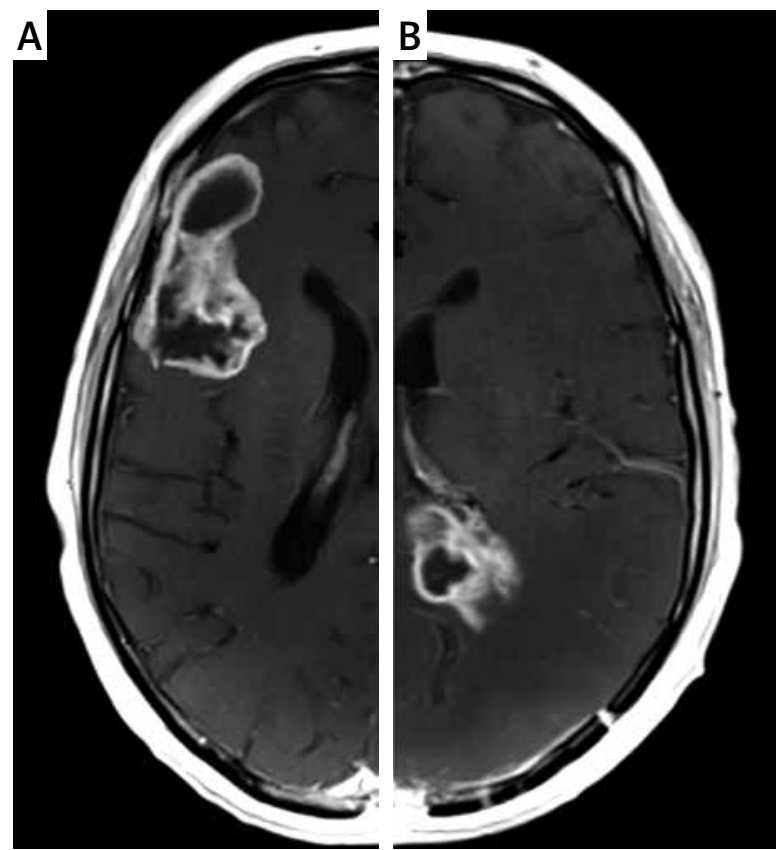

Fig. 4. MRI T1 weighted sequence after Gd-DTPA infusion shows the spatial relationship of glioblastomas related to the SVZ. A) SVZ III tumors involve only the cortex; B) SVZ II contacts only the SVZ.

type II GBs when compared with SVZ type III GBs. Univariate, multivariate, and Kaplan-Meier analyses demonstrated that expression of YKL-40 was a significant negative indicator of the behavior of $G B$.

The present study was performed on a heterogeneous population of GB patients, all of whom underwent total resection, subtotal resection or biopsy followed by the same adjuvant therapy (chemotherapy and radiotherapy). To date, the extent of resection has been an accepted prognostic factor [3,23,33]. Nevertheless, although it is well accepted that tumor resection may improve the symptoms we have found impact on OS only in the univariate analysis. However, extensive resection combined with adjuvant therapy could also explain the advantageous impact in terms of OS in a multivariate analysis.

The difference in survival among patients with GB has been seen to significantly depend on age, KPS at 3 months, extent of resection, and immunoexpression of YKL-40. We have found that there was a significant correlation between the expression of Olig2 and KPS at 3 months. Age at diagnosis and preoperative KPS score have been the most recognized predictors of OS [4]. KPS score at 3 months from diagnosis was a prognostic factor more valuable than KPS 
score at diagnosis, which may be attributed to the influence of surgery. Most of YKL-40+ GBs had a PFS of less than 54 weeks. These data differ from the previous report [2] that found no prognostic association between YKL-40 expression and PFS. However, such disagreement may be because our study has a larger sample size and different age groups of patients.

\section{Conclusions}

Up to date, significant progress has been made in the understanding of GB regarding its topographical molecular expressions. The presence of GBs, which express prognostic markers in relationship to the subventricular zone, is of practical as well as theoretical interest. In fact, this information may be the road by which the most effective therapy can be focused. Our results demonstrated that dismal prognosis of GBs is significantly correlated to YKL-40 expression and linked to SVZ relationship. As YKL-40 has been found in serum and in brain tumor tissue, it has a potential as a therapeutic and prognosis marker for GB. Current insights will ultimately lead to a more individualized therapy for GB patients.

Our study suffers from the same limitations as other retrospective studies, with a biased selection of SVZ topographical locations, which influences the results. Therefore, further controlled studies are needed to validate our results in a prospective study with a greater number of GBs patients.

\section{Disclosure}

\section{Authors report no conflict of interest.}

\section{References}

1. Agnihotri S,Burrell KE, Wolf A, Jalali S, Hawkins C, Rutka JT, Zadeh G. Glioblastoma, a briefreview of history, molecular genetics, animal models and novel therapeuticstrategies. Arch Immunol Ther Exp 2013; 61: 25-41.

2. Antonelli M, Buttarelli FR, Arcella A, Nobusawa S, Donofrio V, Oghaki H, Giangaspero F. Prognostic significance of histological grading, p53 status, YKL-40 expression, and IDH1 mutations in pediatrichigh-grade gliomas. J Neurooncol 2010; 99: 209-215.

3. Bloch O, Han SJ, Cha S, Sun M, Aghi MK, McDermott MW, Berger MS, Parsa A. Impact of extent of resection for recurrent glioblastoma on overall survival. J Neurosurg 2012; 117: 1032-1038.

4. Bozdag S, Li A, Riddick G, Kotliarov Y, Baysan M, Iwamoto FM, Cam MC, Kotliarova S, Fine HA. Age-Specific Signatures of Glioblastoma at the Genomic, Genetic, and Epigenetic Levels. PLos One 2013; 8: e62982.

5. Chaichana KL, Pendleton C, Chambless L, Camara-Quintana J, Nathan JK, Hassam-Malani L, Li G, Harsh IV GR, Thompson RC,
Lim M, Quinones-Hinojosa A. Multi-institutional validation of a preoperative scoring system which predicts survival for patients with glioblastom. J Clin Neurosci 2013; 20: 1422-1426.

6. Chen J, Li Y, Yu TS, McKay RM, Burns DK, Kernie SG, Parada LF. A restricted cell population propagates glioblastoma growth after chemotherapy. Nature 2012; 488: 522-526.

7. Cheng L, Bao S, Rich JN. Potential therapeutic implications of cancer stem cells in glioblastoma. Biochem Pharmacol 2010; 80: 654-665.

8. Colman H, Zhang L, Sulman EP, McDonald M, Shooshtari NL, Rivera a, Popoff S, Nutt CL, Louis DN, Cairncross JG, Gilbert MR, Phillips HS, Mehta MP, Chakravarti A, Pelloski CE, Bhat K, Feuerstein BG, Jenkins RB, Aldape K. A multigene predictor of outcome in glioblastoma. Neurooncol 2010; 12: 49-57.

9. Dahlrot RH, Kristensen BW, Hjelmborg J, Herrstedt J, Hansen S. A population-basedstudy of high-grade gliomas and mutated isocitrate dehydrogenase 1. Int J Clin Exp Pathol 2013; 6: 31-40.

10. Francescone RA, Scully S, Faibish M, Taylor SL, Oh D, Moral L, Yan W, Bentley B, Shao R. Role of YKL-40 in the angiogenesis, radioresistance, and progression of glioblastoma. J Biol Chem 2011; 286: 15332-15343.

11. Georgieva L, Moskvina V, Peirce T, Norton N, Bray NJ, Jones L, Holmans P, MacGregor S, Zammit S, Wilkinson J, Williams $H$, Nikolov I, Williams N, Ivanov D, Davis KL, Haroutunian V, Buxbaum JD, Craddock N, Kirov G, Owen MJ, O’Donovan MC. Convergent evidence that oligodendrocyte lineage transcription factor 2 (OLIG2) and interacting genes influence susceptibility to schizophrenia. Proc Natl Acad Sci USA 2006; 103: 1246912474.

12. Ghazi SO, Stark M, Zhao Z, Mobley BC, Munden A, Hover L, Abel TW. Cell of Origin Determines Tumor Phenotype in anOncogenic Ras/p53 Knockout Transgenic Model of High-Grade Glioma. J Neuropathol Exp Neurol 2012; 71: 729-740.

13. Haskins WE, Zablotsky BL, Foret MR. Molecular characteristics in MRI-classifiedgroup 1 glioblastoma multiforme. Front Oncol 2013; 3: 1-8.

14. Horbinski C, Wang G, Wiley CA. YKL-40 is directly produced by tumor cells and is inversely linked to EGFR in glioblastomas. Int J Clin Exp Pathol 2010; 3: 226-237.

15. Ihrie RA, Alvarez-Buylla A. Lake-front property: a unique germinal niche bythe lateral ventricles of the adult brain. Neuron 2011; 70: 674-686.

16. Iwamoto FM, Hottinger AF, Karimi S, Riedel E, Dantis J, Jahdi M, Panageas KS, Lassman AB, Abrey LE, Fleisher M, DeAngelis LM, Holland EC, Hormigo A. Serum YKL-40 is a marker of prognosis and disease status in high-grade gliomas. Neurooncol 2011; 13 : 1244-1251.

17. Jafri NF, Clarke JL, Weinberg V, Barani IJ, Cha S. Relationship of glioblatoma multiforme to the subventricular zone is associated with survival. Neurooncol 2013; 15: 91-96.

18. Karsy M, Gelbman M, Paarth S, Balumbu O, Moy F, Arslan E. Established and emergingvariants of glioblastoma multiforme: review of morphological and molecular features. Folia Neuropathol 2012; 50: 301-321.

19. Ku BM, Lee YK, Ryu J, Jeong JY, Choi J, Eun KM, Shin HY, Kim DG, Hwang EM, Yoo JC, Park JY, Roh GS, Kim HJ, Cho GJ, Choi WS, 
Paek SH, Kang SS. CHI3L1 (YKL-40)is expressed in human gliomas and regulates the invasion, growth and survival of glioma cells. Int J Cancer 2011; 128: 1316-1326.

20. Ligon KL, Huillard E, Mehta S, Kesari S, Liu H, Alberta JA, Bachoo RM, Kane M, Louis DN, DePinho RA, Anderson DJ, Stiles CD, Rowitch DH. Olig2-regulated lineage-restricted pathway controls replication competence in neural stem cells and malignant glioma. Neuron 2007; 53: 503-517.

21. Lim DA, Cha S, Mayo MC, Chen M, Keles E, VandenBerg S, Berger M. Relationship of glioblastoma multiforme to neural stem cell regions predicts invasive and multifocal tumor phenotype. Neurooncol 2007; 9: 424-429.

22. Marshall CAG, Novitch BG, Goldman JE. Olig2 Directs Astrocyte and Oligodendrocyte Formation in Postnatal Subventricular Zone Cells. J Neurosci 2005; 25: 7289-7298.

23. McGirt MJ, Chaichana KL, Attenello FJ, Weingart JD, Than K, Burger PC, Olivi A, Brem H, Quinones-Hinojosa A. Extent of surgical resection is independently associated with survival in patients with hemispheric infiltrating low-grade gliomas. Neurosurgery 2008; 63: 700-708.

24. Michaelsen SR, Christensen IJ, Grunnet K, Stockhausen M, Broholm H, Kostaljanetz M, Poulsen HS. Clinical variables serve as prognostic factors in a model for survival from glioblastoma multiforme: an observational study of a cohort of consecutive non-selected patients from a single institution. BMC Cancer 2013; 13: 1-11

25. Park CK, Kim JH, Nam DH, Kim CY, Chung SB, Kim YH, Seol HJ, Kim TM, Choi SH, Lee SH, Heo DS, Kim IH, Kim DG, Jung HW. A practical scoring system to determine whether to proceed with surgical resection in recurrent glioblastoma. Neurooncol 2013; 15: 1096-1101.

26. Pelloski CE,Mahajan A, Maor M, Chang EL, Woo S, Gilbert M, Colman H, Yang H, Ledoux A, Blair H, Passe S, Jenkins RB, Aldape KD. YKL-40 Expression is Associated with Poorer Response to Radiation and Shorter OverallSurvival in Glioblastoma. Clin Cancer Res 2005; 11: 3326-3334.

27. Piña-Batista KM, Fernandez-Vega I, deEulate-Beramendi SA, Gutierrez-Morales JC, Kurbanov A, Asnel D, Meilán A, AstudiIlo A. Prognostic significance of the markers IDH1 and YKL40 related to the subventricular zone. Folia Neuropathol 2015; 53: 52-59.

28. Reya T, Morrison SJ, Clarke MF, Weissman IL. Stem cells, cancer, and cancer stem cells. Nature 2001; 414: 105-111.

29. Schiffer D, Mellai M, Annovazzi L, Caldera V, Piazzi A, Denysenko T, Melcarne A. Stem cell niches in glioblastoma: a neuropathological view. Biomed Res Int 2014: 2014: 1-7.

30. Shao R, Francescone RA, Ngernyuang N, Bentley B, Taylor SL, Moral L, Yan W. Anti-YKL-40 antibody and ionizing irradiation synergistically inhibit tumor vascularization and malignancy in glioblastoma. Carcinogenesis 2014; 35: 373-382.

31. Stupp R, Mason WP, van den Bent MJ, Weller M, Fisher B, Taphoorn MJ, Belanger K, Brandes AA, Marosi C, Bogdahn U, Curschmann J, Janzer RC, Ludwin SK, Gorlia T, Allgeier A, Lacombe D, Cairncross JG, Eisenhauer E, Mirimanoff RO; European Organisation for Research and Treatment of Cancer Brain Tumor and Radiotherapy Groups; National Cancer Institute of Canada Trials Group. Radiotherapy plus concomitant and adju- vant temozolomide for glioblastoma. N Engl J Med 2005; 352: 987-996.

32. Tabu K, Ohba Y, Suzuki T, Makino Y, Kimura T, Ohnishi A, Sakai M, Watanabe T, Tanaka S, Sawa H. Oligodendrocyte lineage transcription factor 2 inhibits the motility of a human glial tumor cell line by activating RhoA. Molecular Cancer Res 2007; 5: 1099-1109.

33. Urbanska K, Sokolowska J, Szmidt M, Sysa P. Glioblastoma multiforme- an overview. Contemp Oncol 2014; 18: 307-312.

34. Van Meir EG, Hadjipanayis CG, Norden AD, Shu HK, Wen PY, Olson JJ. Exciting new advances in neuro-oncology: the avenue to a cure for malignant glioma. Cancer J Clin 2010; 60: 166-193.

35. Yip S, Butterfield YS, Morozova O, Chittaranjan S, Blough MD, An J, Birol I, Chesnelong C, Chiu R, Chuah E, Corbett R, Docking R, Firme M, Hirst M, Jackman S, Karsan A, Li H, Louis DN, Maslova A, Moore R, Moradian A, Mungull KL, Perizzolo M, Qian J, Roldan G, Smith EE, Tamura-Wells J, Thiessen N, Varhol R, Weiss S, Wu W, Young S, Zhao Y, Mungall AJ, Jones SJ, Morin GB, Chan JA, Caincross JG, Marra MA. Concurrent CIC mutations, IDH mutations and $1 p / 19 q$ loss distinguish oligodendrogliomas from other cancers. J Pathol 2012; 226: 7-16.

36. Zhang W, Kawanishi M, Miyake K, Kagawa M, Kawai N, Murao K, Nishiyama A, Fei Z, Zhang X, Tamiya T. Associationbetween YKL-40 and adult primary astrocytoma. Cancer 2010; 116: 2688-2697.

37. Zhu VF, Yang J, Lebrun DG, Li M. Understanding the role of cytokines in Glioblastoma Multiforme pathogenesis. Cancer Lett 2012; 316: 139-150. 